\title{
Engineering Point of View on Liver Transplantation Strategies: Multi-Level Modeling of Hepatic Perfusion
}

\author{
C. Debbaut ${ }^{\mathrm{a}, \star}$, D. Monbaliu ${ }^{\mathrm{b}}$, and P. Segers ${ }^{\mathrm{a}}$
}

aBiofluid, Tissue and Solid Mechanics for Medical Applications (bioMMeda), Department of Electronics and Information Systems, iMinds Medical IT Department, Ghent University, Ghent, Belgium; and ${ }^{\mathrm{b}}$ Abdominal Transplant Surgery, University Hospitals Leuven, and Department of Microbiology and Immunology, KU Leuven, Leuven, Belgium

\begin{abstract}
Background. Hepatic perfusion plays a crucial role in liver transplantation strategies, for example, when preserving procured organs with the use of machine perfusion preservation (MP) and in the case of living donor liver transplantation (LDLT). Liver hemodynamics are not yet fully understood because of insufficient knowledge on the hepatic vascular morphology and its perfusion characteristics, hampering the optimization of liver transplantation procedures. To this end, we developed computer models to simulate the complex blood circulation through the liver from the macro-scale down to the terminal micro-scale level.

Methods. A combination of state-of-the-art techniques (vascular corrosion casting, microCT scanning up to a $2.6-\mu \mathrm{m}$ resolution, and image processing) led to $3 \mathrm{D}$ visualizations and detailed geometrical analyses of the complex architecture of the liver's 3 vascular trees, ranging from the largest vessels (macrocirculation) down to the sinusoids (microcirculation).

Results. On the basis of these data, we developed various computational models (electrical analog models and 3D computational fluid dynamics models) to study the blood flow-induced forces acting on the hepatic blood vessels. The latter was done for physiological blood flow through the liver as well as for livers undergoing MP or LDLT procedures. Hereby, several scenarios were simulated to study the behavior of livers in different hemodynamic circumstances.

Conclusions. A novel, multi-level modeling framework was developed to simulate hepatic perfusion in support of liver transplantation strategies. We obtained unique anatomical data on the vascular architecture of both human and rat livers. These data formed the building blocks of electrical analog models of hepatic perfusion and numerical models of the liver microcirculation. The results revealed novel insights into the hemodynamic impact of liver MP and LDLT procedures as well as into the microcirculatory perfusion characteristics. The presented methodology is also applicable to other tree-like structures (eg, the biliary tree) or organs (eg, kidneys, lungs).
\end{abstract}

\begin{abstract}
$\mathbf{A}^{\mathrm{t}}$ LTHOUGH liver transplantation has become a successful treatment for liver failure, a substantial lack of suitable donor livers has arisen during the past decades. This shortage urged the expansion of the donor pool to reduce the waiting lists themselves as well as the waiting list mortality. One of the options to enlarge the donor pool is the usage of high risk liver grafts, originating from donation after circulatory death or other extended criteria donors. These organs are typically more susceptible to ischemia-reperfusion injury (partly worsened by static cold storage) than standard criteria
\end{abstract}

livers [1]. Hence, machine perfusion (MP) was put forward as an alternative preservation technique to allow for better and longer preservation than cold storage. Experimental studies showed better organ viability after MP, especially for extended criteria livers [2]. However, potential risks of liver MP include flow competition between the hepatic arterial

*Address correspondence to Charlotte Debbaut, IBiTech-bioMMeda, Ghent University, Campus Heymans-Blok B, De Pintelaan 185, 9000 Gent, Belgium. E-mail: charlotte.debbaut@ugent.be

$0041-1345 / 14$ 
1. Hypothermic machine perfusion (HMP) experiment of a human liver [7]
2. Vascular corrosion casting and dissection of a mesocirculation and microcirculation sample [8]

3. High resolution micro-CT scanning and image processing resulting in $3 D$ reconstructions [8]
4. Development of computer models to simulate liver perfusion [9-11]
A Schematic illustration of HMP setup

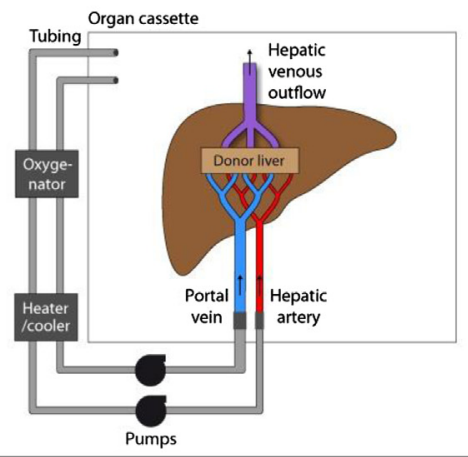

B Human liver HMP experiment

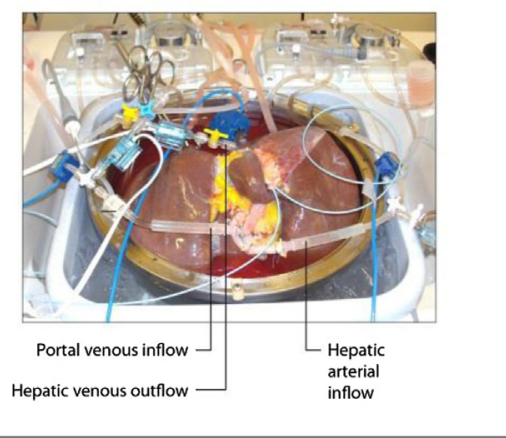
$\downarrow$
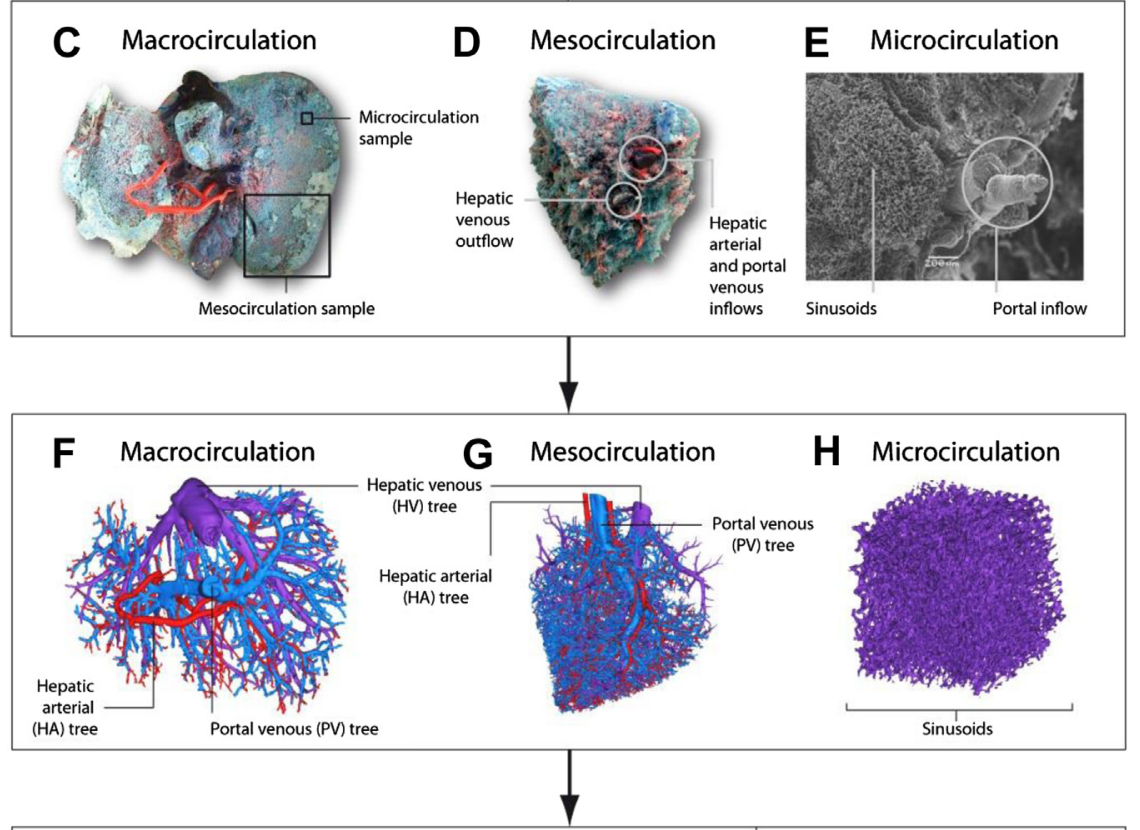

I Electrical analog model of human liver perfusion [9]

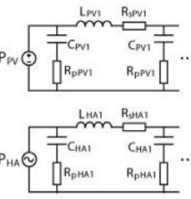

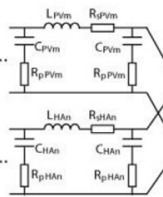

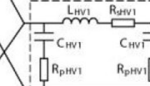

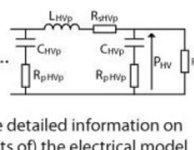
See [9] for more detailed information on

J Results of the electrical analog model: pressure distribution and sinusoidal shear stresses for normal blood flow and HMP at physiological or low pressure settings [9]
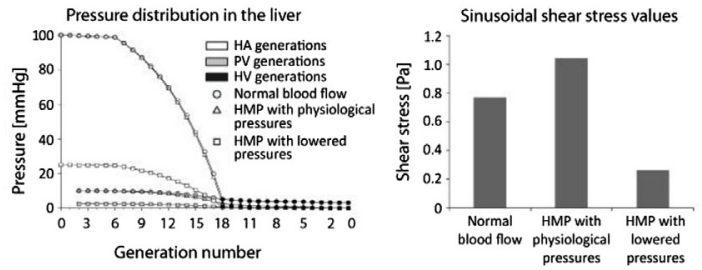

K CFD model of terminal liver microcirculation [10]

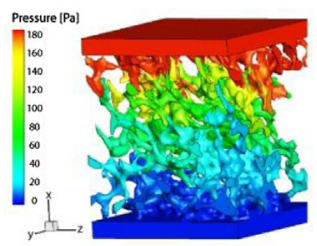

L Porous medium CFD model of a lobule [11]

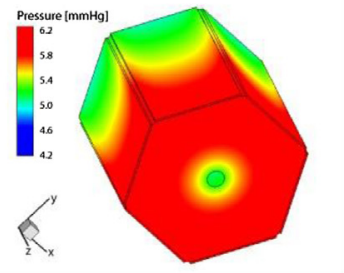


(HA) and portal venous (PV) inflow [3] as well as endothelial cell injury [4]. Therefore, a profound knowledge of the optimal perfusion settings is needed to guarantee sufficient and homogeneous liver perfusion while preventing microcirculatory damage.

A second option to overcome the organ shortage is the use of alternative transplantation techniques such as split liver (SLT) and living donor liver transplantation (LDLT). Although promising, the main drawback of these techniques is the risk for developing the small-for-size syndrome, which may lead to severe complications and even graft failure [5]. A more elaborate insight in liver hemodynamics is thus necessary to determine the optimal location and amount of liver tissue to resect.

Because hepatic perfusion plays a crucial role in the transplantation strategies mentioned above, our objective was to model the liver blood circulation at multiple length scales to investigate the hemodynamic impact of MP and SLT or LDLT procedures [6].

\section{METHODS}

Hypothermic MP (HMP) experiments were conducted with the LifePort Workstation (Organ Recovery Systems, Zaventem, Belgium) on human liver grafts that were discarded for transplantation after failed reallocation (Fig 1A,B). The protocol was approved by the Ethics Committee of the University Hospitals Leuven (Leuven, Belgium) and by the Belgian Liver and Intestine Committee and was in accordance with the Helsinki Declaration of 1975 (as revised in 1983). The HMP experiments allowed measurement of liver perfusion behavior for different settings (eg, varying pressures) [7]. After HMP, the liver circulation was materialized by means of vascular corrosion casting (Batson's No. 17, Polysciences, Warrington, Pa, United States), resulting in a replica of the hepatic vasculature (Fig 1C). Micro-CT scanning (UGCT, Ghent University, Gent, Belgium) and image processing (Mimics, Materialise, Leuven, Belgium) enabled visualizing the replica in globo $( \pm 110 \mu \mathrm{m}$ resolution; macrocirculation). To study the blood vessels distal to the macrocirculation, mesocirculation and microcirculation samples (Fig 1D-E) were consecutively dissected from the replica and visualized at a $71 \mu \mathrm{m}$ and $2.6 \mu \mathrm{m}$ resolution, respectively. The resulting $3 \mathrm{D}$ reconstructions (Fig $1 \mathrm{~F}-\mathrm{H}$ ) enabled a thorough quantitative analysis of branching patterns and geometric features (vessel radii and lengths) of the vascular trees. More detailed information on these methods is available [8].

On the basis of the morphological macrocirculation and mesocirculation data of the human liver and extrapolations thereof, an electrical analog model of the human intrahepatic hemodynamics was established (Fig 1I). This approach builds on the analogy of blood flow through a vessel and the electrical current through a circuit. The resulting model was implemented in Matlab (MathWorks, Natick, Mass, United States) and enables simulating pressure and flow parameters throughout the liver vasculature for different boundary conditions corresponding to natural blood flow or MP (see Debbaut et al [9] for more information). With the use of the experimentally acquired HMP data (see previous paragraph; Fig 1A,B), HA and PV input impedances were calculated to validate and calibrate the model to a specific liver [7].

At the microcirculatory level, structural-related perfusion characteristics (eg, porosity and permeability) were investigated by means of computational fluid dynamics (CFD) modeling. These models were constructed using 3D reconstructions of the human hepatic microstructure (Fig $1 \mathrm{H})$ to numerically simulate the blood flow through the sinusoids (Fig 1K; see Debbaut et al [10] for more information). The resulting permeability characteristics enabled building a 3D porous medium CFD model of a classic liver lobule (Fig 1L), also known as the hepatic structural unit. This porous medium model was used to study the effect of anisotropic permeability and vascular septa on the microcirculatory flow (see Debbaut et al [11] for more information).

In addition to human livers, rat livers (originating from 10-weeksold female Wistar rats weighing 200-225 g) were used to study the hemodynamic impact of partial hepatectomy procedures, such as LDLT. Similar to the methodology for human livers, detailed geometric data of rat livers were gathered by combining vascular corrosion casting, micro-CT scanning $( \pm 50 \mu \mathrm{m}$ resolution), and image processing. The resulting $3 \mathrm{D}$ reconstructions enabled performing virtual resections to mimic a $70 \%, 80 \%$, and $90 \%$ partial hepatectomy. A lobe-specific electrical analog model of the rat liver (modification of the electrical model for the human liver; see Debbaut et al [12] for more elaborate information) was developed to study the hemodynamics in normal livers as well as partially hepatectomized livers.

\section{RESULTS AND DISCUSSION}

Detailed 3D reconstructions and unique geometrical data on the vascular architecture of one and the same human liver were obtained at the macrocirculation, mesocirculation, and microcirculation levels (Fig 1F-H). The branching topology of the HA, PV, and hepatic venous vascular trees was analyzed up to 13 blood vessel generations, corresponding to the combined results of the macrocirculation and mesocirculation (Fig 1F,G). Vessel diameters ranged from $26 \mathrm{~mm}$ to $0.16 \mathrm{~mm}$; lengths ranged from $74 \mathrm{~mm}$ to $0.74 \mathrm{~mm}$. Interestingly, the geometric features gave evidence of an exponential relation with the generation number. The number of vessels $(y)$, for instance, rises exponentially with increasing generation numbers $(x)$, resulting in the equation $y=0.4 \cdot e^{x}$ for the HA tree [8]. The microcirculation sample showed a very complex network of interconnected and intertwined sinusoids (Fig $1 \mathrm{H}$; diameters of $\pm 11-15 \mu \mathrm{m}$ ), with an estimated porosity of $14.3 \%$ [10].

Fig 1. This flow chart explains the work flow followed in this study: schematic illustration (A) and illustration (B) of a hypothermic machine perfusion (HMP) experiment with a human liver. Subsequently, vascular corrosion casting resulted in a vascular replica (C), out of which mesovascular (D) and microvascular samples (E; scanning electron microscopy image) were dissected. Micro-CT scanning resulted in $3 \mathrm{D}$ reconstructions $(\mathbf{F}, \mathbf{G}$, and $\mathbf{H})$. On the basis of detailed geometric analyses of the macrocirculatory and mesocirculatory reconstructions, an electrical analog model of human liver perfusion (I) was developed to simulate perfusion parameters such as pressures and sinusoidal shear stresses for different hemodynamic circumstances $(\mathbf{J})$. The perfusion characteristics of the microcirculation sample were investigated by means of 3D computational fluid dynamics (CFD) models, of which a pressure distribution is shown (K). The resulting permeability values were used to build a 3D porous medium CFD model of a liver lobule (L). 
The electrical analog model of human hepatic perfusion reproduced the experimentally observed HA-PV flow competition during pressure-driven HMP, inducing a decreasing PV flow when the HA flow was increasing $[3,9]$. From a biomechanical point of view, the results (Fig 1J) indicate that pressure-controlled HMP with low pressures results in a lower sinusoidal shear stress (estimated value of $0.26 \mathrm{~Pa})$ than normal physiological blood flow $(0.77 \mathrm{~Pa})$ and hence has a lower chance to induce organ damage than HMP at physiological pressures $(1.04 \mathrm{~Pa})$. The perfusion fluid viscosity appears to have no effect on shear stress levels during pressure-driven HMP (in contrast to flow-driven HMP) [9]. These findings imply that the electrical model is potentially applicable to virtually test and compare perfusion conditions. The presented simulations were done for HMP, but model parameters are adjustable to allow simulating (sub)normothermic MP [13].

CFD models of the human hepatic microcirculation indicate an anisotropic behavior in terms of permeability. More specifically, permeability values were higher along the direction of the central vein $\left(3.6 \cdot 10^{-14} \mathrm{~m}^{2}\right)$, while two times lower but approximately equal permeabilities were observed along the radial (Fig $1 \mathrm{~K} ; 1.6 \cdot 10^{-14} \mathrm{~m}^{2}$ ) and circumferential $\left(1.8 \cdot 10^{-14} \mathrm{~m}^{2}\right)$ directions according to a liver lobule [10]. When implementing the resulting permeability values in the porous lobule model (Fig $1 \mathrm{~L}$ ), the inclusion of vascular septa shows a more homogeneous and more physiologically plausible lobule perfusion with better perfusion of the zones in between portal tracts. In addition, our findings show that the lobule model can be calibrated to physiological perfusion parameters [11].

The electrical rat liver model clearly demonstrated hyperperfusion effects such as elevated lobe-specific PV flows of 0.41 to $1.8,1.0$ to $3.0,1.8$ to $3.5,7.4 \mathrm{~mL} / \mathrm{min}$ and increased portal pressures of 5.9, 11, 12, $24 \mathrm{~mm} \mathrm{Hg}$ for $0 \%, 70 \%, 80 \%$, $90 \%$ hepatectomy, respectively. Remarkably, comparison of two $90 \%$ resection techniques demonstrated significantly different results, with, for instance, PV pressures differing $14 \%$ (24 vs $21 \mathrm{~mm} \mathrm{Hg}$ ). It is a plausible hypothesis that the technique leading to the lowest portal hypertension also leads to a better survival [12].

\section{CONCLUSIONS AND FUTURE PERSPECTIVE}

The take-home message of this work is that an innovative multi-level modeling framework has been developed to simulate hepatic perfusion in support of the optimization of liver transplantation strategies [6]. This framework consists of anatomic models of the hepatic vascular architecture, electrical analog models of hepatic perfusion, and numeric models of the hepatic microcirculation. These versatile tools resulted in unique morphological data on human and rat livers and novel insights into the hemodynamic impact of liver MP and SLT or LDLT procedures as well as into the perfusion characteristics of the liver microcirculation. Although a number of missing pieces of the liver perfusion puzzle were collected, further research is necessary to reveal the complete picture (eg, validation of the micromodels, extending the number of studied livers and samples, and extrapolation of the rat model for partial hepatectomy to human livers). In the longer term, future work may contribute to the prediction of liver-specific optimal MP settings and an advanced MP monitoring system, enabling real-time tracking of the organ behavior and automatic feedback control of perfusion parameters. Moreover, the developed methodologies are not only applicable to transplantation-related issues but also to enlighten the underlying disease processes of liver pathologies (eg, cirrhosis) or to help planning patient-specific surgical interventions (eg, placement of a transjugular intrahepatic portosystemic shunt). In addition, the perfusion characteristics of other tree-like structures (eg, the biliary tree) or organs (eg, kidneys or lungs) may be investigated in a similar way.

\section{ACKNOWLEDGMENTS}

This research was supported by the Agency for Innovation by Science and Technology in Flanders, Belgium (IWT; project 101115).

\section{REFERENCES}

[1] Monbaliu D, Pirenne J, Talbot D. Liver transplantation using Donation after Cardiac Death donors. J Hepatol 2012;56:474-85.

[2] Bae C, Henry SD, Guarrera JV. Is extracorporeal hypothermic machine perfusion of the liver better than the 'good old icebox'? Curr Opin Organ Transplant 2012;17:137-42.

[3] Monbaliu D, Debbaut C, Hillewaert W, et al. Flow competition between hepatic arterial and portal venous flow during hypothermic machine perfusion preservation of porcine livers. Int $\mathrm{J}$ Artif Organs 2012;35:119-31.

[4] Jain S, Xu HZ, Duncan H, et al. Ex-vivo study of flow dynamics and endothelial cell structure during extended hypothermic machine perfusion preservation of livers. Cryobiology 2004;48:322-32.

[5] Yagi S, Uemoto S. Small-for-size syndrome in living donor liver transplantation. Hepatobiliary Pancreat Dis Int 2012;11:570-6.

[6] Debbaut C. Multi-Level Modelling of Hepatic Perfusion in Support of Liver Transplantation Strategies. PhD dissertation. Gent, Belgium: Ghent University; 2013.

[7] Debbaut C, Monbaliu D, Segers P. Validation and tuning of an electrical analog model of human liver perfusion based on hypothermic machine perfusion experiments. Int J Artif Organs 2014;37(6):486-98.

[8] Debbaut C, Segers P, Cornillie P, et al. Analyzing the human liver vascular architecture by combining vascular corrosion casting and micro-CT scanning: a feasibility study. J Anat 2014;224:509-17.

[9] Debbaut C, Monbaliu D, Casteleyn C, et al. From vascular corrosion cast to electrical analog model for the study of human liver hemodynamics and perfusion. IEEE Trans Biomed Eng 2011;58:25-35.

[10] Debbaut C, Vierendeels J, Casteleyn C, et al. Perfusion characteristics of the human hepatic microcirculation based on three-dimensional reconstructions and computational fluid dynamic analysis. J Biomech Eng 2012;134:011003.

[11] Debbaut C, Vierendeels J, Siggers J, et al. A 3D porous media liver lobule model: the importance of vascular septa and anisotropic permeability for homogeneous perfusion. Comput Methods Biomech Biomed Eng 2014;17:1295-310.

[12] Debbaut C, De Wilde D, Casteleyn C, et al. Modeling the impact of partial hepatectomy on the hepatic hemodynamics using a rat model. IEEE Trans Biomed Eng 2012;59:3293-303.

[13] Hessheimer AJ, Fondevila C, Garcia-Valdecasas JC. Extracorporeal machine liver perfusion: are we warming up? Curr Opin Organ Transplant 2012;17:143-7. 